CARTA AL EDITOR

\section{DERIVADOS DE HIPROMELOSA PARA PREVENIR LA INFECCIÓN POR EL SARS-COV-2: UNA REVISIÓN SISTEMÁTICA}

\section{HYPROMELLOSE DERIVATIVES FOR PREVENTING SARS-COV-2 INFECTION: A SYSTEMATIC REVIEW}

\author{
Akram Hernández-Vásquez (1D) 1,a, Diego Azañedo (1D) 2,b, \\ Rodrigo Vargas-Fernández (iD) $2, c$, \\ Daniel Comandé (iD ${ }^{3, d}$
}

\footnotetext{
1 Universidad San Ignacio de Loyola, Vicerrectorado de Investigación, Centro de Excelencia en Investigaciones Económicas y Sociales en Salud, Lima, Perú.

2 Universidad Científica del Sur, Lima, Perú.

3 Instituto de Efectividad Clínica y Sanitaria (IECS), Buenos Aires, Argentina.

a Médico cirujano, magíster en Gestión y Políticas Públicas; ${ }^{\text {b }}$ cirujano dentista, magíster en Estomatología; ${ }^{\mathrm{c}}$ médico cirujano; ${ }^{\mathrm{d}}$ licenciado en Bibliotecología y Ciencia de la Información.
}

Sr. Editor: En el Perú, la enfermedad por coronavirus (COVID-19) ha generado una de las tasas de mortalidad más altas en el mundo ${ }^{(1)}$. Parte de este problema se puede fundamentar en que se decidió implementar algunas medidas terapéuticas en ausencia de evidencia sobre su eficacia y seguridad, y solo con base en la opinión de expertos ${ }^{(2)}$. Posteriormente, con la disponibilidad de ensayos clínicos aleatorizados sobre el uso de medicamentos contra la COVID-19, muchos de ellos han sido retirados de las recomendaciones de tratamiento en el Perú, ya sea ante la ausencia de algún beneficio clínico o por haber agravado el pronóstico del paciente, en comparación con la mejor terapia de soporte ${ }^{(2)}$. Recientemente, un derivado en polvo de la hipromelosa (como el producto Taffix) ha sido promocionado como una medida preventiva contra la COVID-19 mediante el bloqueo de los virus dentro de la cavidad nasal ${ }^{(3)}$; sin embargo, la hipromelosa en solución es empleada comúnmente para tratar los síntomas de sequedad ocular ${ }^{(4)}$. Por ello, la presente revisión sistemática tuvo como objetivo determinar el posible efecto de los derivados de hipromelosa en la prevención contra la infección por el SARS-CoV-2.

\footnotetext{
Citar como: Hernández-Vásquez A, Azañedo D, Vargas-Fernández R, Comandé D. derivados de hipromelosa para prevenir la infección por el SARS-CoV-2: una revisión sistemática. Rev Peru Med Exp Salud Publica. 2021;38(3):469-71. doi: https://doi.org/10.17843/rpmesp.2021.383.9116
}

Correspondencia: Akram Abdul Hernández Vásquez; Universidad San Ignacio de Loyola, Av. La Fontana 550, La Molina, Lima, Perú; ahernandez@usil.edu.pe

Recibido: 25/07/2021 Aprobado: 29/09/2021 En línea: 31/09/2021
El protocolo de la revisión sistemática fue registrado en PROSPERO (Registro Internacional Prospectivo de Revisiones Sistemáticas) con el número de referencia CRD42021269359. Se buscaron estudios que evaluaron la eficacia, efectividad o seguridad del uso de derivados de hipromelosa en la prevención contra la infección por el SARS-CoV-2. La búsqueda electrónica se realizó en PubMed, Cochrane Library, Embase, CINAHL, Web of Science, Scopus, LILACS, SciELO, medRxiv, bioRxiv, ClinicalTrials.gov y Google Académico hasta el 22 de julio de 2021 y se actualizó hasta el 24 de setiembre de 2021. Los detalles de las búsquedas, recolección, selección, criterios de inclusión y evaluación para el desarrollo de la revisión sistemática se incluyen en el Material suplementario. Como resultado de la búsqueda se identificaron un total de 21 registros luego de la eliminación de duplicados. Luego de la evaluación por títulos y resúmenes, ninguno de los estudios o registros de estudios identificados cumplieron los criterios de inclusión para la presente revisión sistemática (Figura 1).

Es importante mencionar que, antes de realizar esta revisión sistemática, en una revisión narrativa de la evidencia realizada por una institución española, se evaluó el uso de Taffix como tratamiento preventivo contra la COVID-19, basándose en la mejor evidencia disponible ${ }^{(5)}$. En esta revisión se describió un estudio prospectivo que evaluó el uso de los derivados de hipromelosa como medida preventiva contra la infección por el SARS-CoV-2, y se consideró que, sobre la base de ese único estudio no podría recomendarse el uso de los derivados de hipromelosa en la prevención contra la COVID-19 ${ }^{(6)}$. Dicho estudio también fue identificado durante el proceso de nuestra búsqueda sistemática y fue parte de los 21 registros cribados para evaluar su elegibilidad, sin embargo, este no fue incluido debido a que, los autores del estudio determinaron la presencia de la infección por el SARS-CoV-2 en la muestra seleccionada mediante una encuesta telefónica. El estudio en mención reportó que el uso de este fármaco se asoció con una reducción del 78\% (intervalo de confianza [IC] 95\%: 1\%-95\%) en el odds de infectarse con el SARS-CoV-2. Al respecto, se deben mencionar las principales limitaciones de este estudio como la falta de aleatorización de los participantes a los grupos que utilizaron o no el fármaco. Esto no permite el control adecuado de confusores en el cálculo del estimador del efecto del fármaco sobre el desenlace de interés, más aún cuando no se tomaron en cuenta a factores de confusión relevantes, como por ejemplo, uso de mascarillas, contacto directo con personas positivas, entre otros. De igual manera, la definición de caso de infección por SARS-CoV-2 se basó en el autorreporte del paciente realizado a través de una entrevista telefónica, sin mencionar si se verificó la existencia de dicha infección en los registros médicos del paciente. Asimismo, la asociación reportada por los autores posee un IC muy amplio (entre $1 \%$ y 95\%), lo que es señal de falta de precisión del resultado, probablemente debido al escaso tamaño de la muestra. Por último, no se mencionó si el estudio contaba con la aprobación de un comité de ética. Estas y otras limitaciones 
Identificación de nuevos estudios en bases de datos y registros

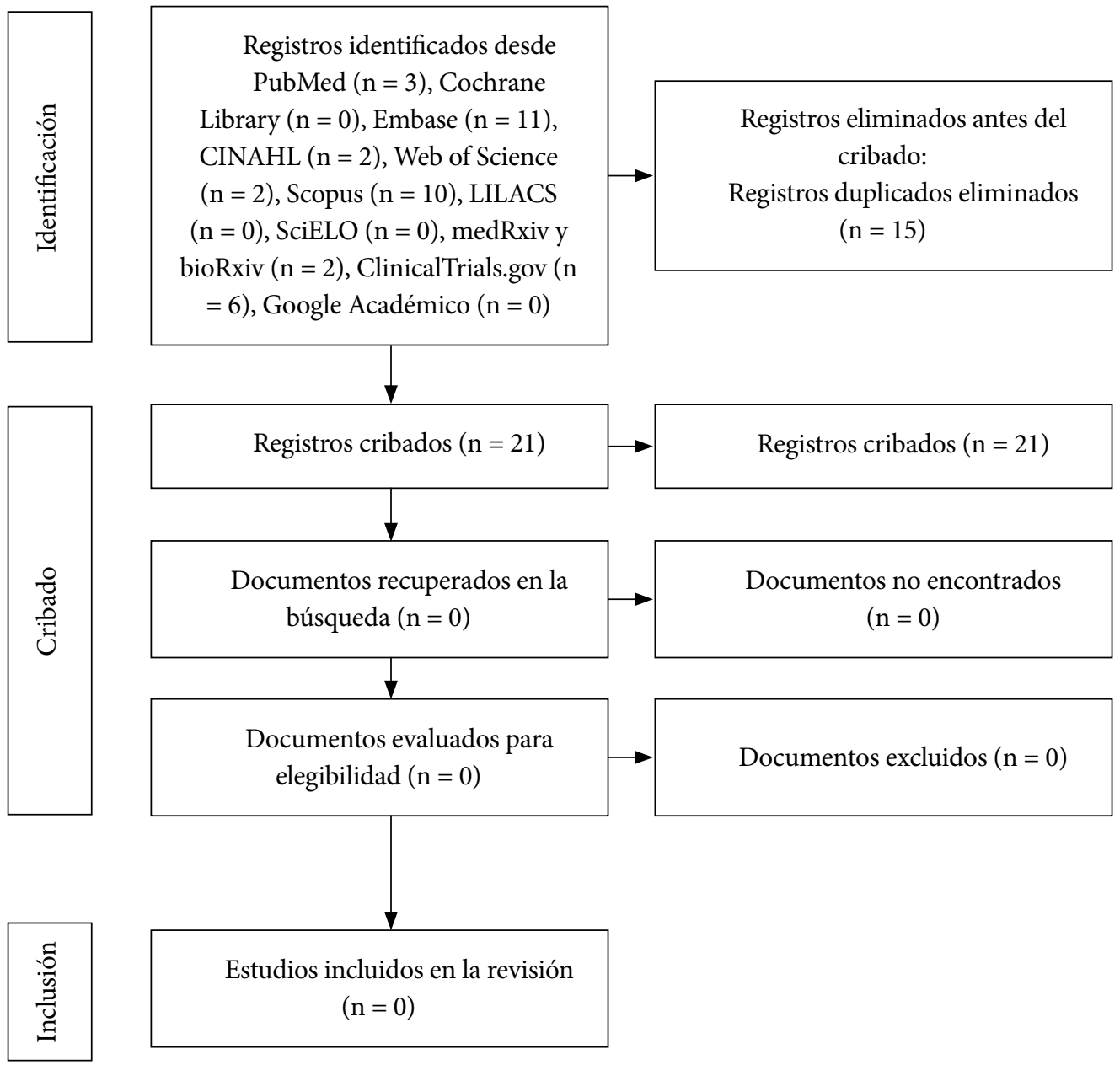

Figura 1. Flujo de la selección de estudios según la declaración PRISMA.

Fuente: Basado en Page MJ, McKenzie JE, Bossuyt PM, Boutron I, Hoffmann TC, Mulrow CD, et al. The PRISMA 2020 statement: an updated guideline for reporting systematic reviews. BMJ 2021;372:n71. doi: 10.1136/bmj.n71

restan confiabilidad a los resultados de este trabajo. Se debe precisar que es justamente este estudio en el que los productores de Taffix sustentan su recomendación de uso para prevenir la infección por el SARS-CoV-2 ${ }^{(3)}$.

En conclusión, no existe evidencia clínica que respalde el uso de los derivados de hipromelosa como medida preventiva contra la infección por el SARS-CoV-2. Por ello, es responsabilidad de los profesionales de la salud ser prudentes al momento de emitir una recomendación sobre medicamentos sin eficacia y seguridad comprobada; esta responsabilidad debe ser aún mayor en aquellos profesionales que cuentan con reconocimiento mediático y cuyas opiniones puedan tener un mayor impacto en la salud pública. Enfatizamos en que la promoción del uso de productos farmacológicos contra la COVID-19 con beneficio incierto podría generar una falsa sensación de protección entre la población, contribuyendo al incremento en la incidencia de casos y muertes por la COVID-19 en nuestro país.

Contribuciones de los autores: AHV, DA, RVF y DC participaron en la concepción del estudio, la redacción y aprobación de la versión final del manuscrito; y asumen responsabilidad de su contenido. DC realizó las búsquedas bibliográficas.

Fuentes de financiamiento: Autofinanciado.

Conflictos de interés: AHV es miembro del comité editor de la Revista Peruana de Medicina Experimental y Salud Pública. Los demás autores declaran no tener conflicto de interés alguno.

Material suplementario: Disponible en la versión electrónica de la RPMESP. 


\section{REFERENCIAS BIBLIOGRÁFICAS}

1. Dyer O. Covid-19: Peru's official death toll triples to become world's highest. BMJ. 2021;373:n1442. doi: 10.1136/bmj.n1442.

2. Solari L.El 2021 y sus nuevos retos en el control del SARS-CoV-2. Rev Peru Med Exp Salud Publica. 2021;38(1):5-6. doi: 10.17843/ rpmesp.2021.381.7312.

3. Taffix $\mathrm{x}^{\mathrm{TM}}$ - Nasus Pharma [Internet]. [citado el 24 de julio de 2021]. Disponible en: https://www.nasuspharma.com/taffix/

4. Doughty MJ, Glavin S. Efficacy of different dry eye treatments with artificial tears or ocular lubricants: a systematic review.
Ophthalmic Physiol Opt. 2009;29(6):573-83. doi: 10.1111/j.14751313.2009.00683.x.

5. Revisión sobre la evidencia científica del spray nasal: Taffix ${ }^{\infty}$ SEORL-CCC [Internet]. [citado el 24 de julio de 2021]. Disponible en: https://seorl.net/revision-cientifica-spray-nasal-covid/

6. Shmuel K, Dalia M, Tair L, Yaakov N. Low pH Hypromellose (Taffix) nasal powder spray could reduce SARS-CoV-2 infection rate post mass-gathering event at a highly endemic community: an observational prospective open label user survey. Expert Rev Anti Infect Ther. 2021;1-6. doi: 10.1080/14787210.2021.1908127. 